\title{
Caracterização Morfológica de espécies de Trapoeraba (Commelina spp.) UTILIZANDO A ANÁlise MUlTIVARIAdA ${ }^{1}$
}

\author{
Morphological Characterization of Wandering-Jew Species (Commelina spp.) Using \\ Multivariate Analysis
}

ROCHA, D.C. ${ }^{2}$, RODELLA, R.A. ${ }^{3}$ e MARTINS D. ${ }^{4}$

\begin{abstract}
RESUMO - As espécies Commelina benghalensis, C. villosa, C. diffusa e C. erecta são conhecidas como trapoeraba e, freqüentemente, são confundidas entre si, dificultando o controle químico, o que pode provocar prejuízos econômicos e danos ambientais. O presente trabalho teve como objetivo selecionar características morfológicas que possibilitem facilitar a identificação dessas espécies, utilizando a técnica de análise multivariada. Foram avaliadas 12 características morfológicas descritivas e 13 quantitativas, utilizando-se os métodos de análise de agrupamento e análise de componentes principais. As espécies apresentaram alto grau de dissimilaridade, sobretudo em relação às características descritivas, destacandose: hábito da planta, pilosidade do caule e da folha, entre outros. As características quantitativas também mostraram poder discriminatório. Características que apresentaram alto valor taxonômico foram selecionadas para compor a chave de identificação para as quatro espécies de Commelina.
\end{abstract}

Palavras-chave: Commelinaceae, morfologia vegetal, taxonomia vegetal.

\begin{abstract}
Commelina benghalensis, C. villosa, C. diffusa and C. erecta are known as wandering-jew in Brazil and are frequently misidentified, causing unsatisfactory herbicide control and promoting economic losses and environmental damage. This work aimed to select morphological characteristics of these four types of weeds using multivariate analysis methods to facilitate their identification. Twelve descriptive and thirteen quantitative morphological characteristics were used in clustering analysis and principal component analysis. Commelina species presented a high degree of dissimilarity, especially in their descriptive characteristics, such as, plant habit, stem and leaf pilosity. Quantitative characteristics also showed high differentiation degree. Morphological descriptive, and quantitative characteristics with taxonomic importance were selected to propose identification key for the four weed species.
\end{abstract}

Keywords: Commelinaceae, plant morphology, plant taxonomy.

1 Recebido para publicação em 23.4.2007 e na forma revisada em 29.11.2007.

Extraído do capítulo I da tese de doutorado da primeira autora.

2 Professora Adjunta do Dep. de Biologia Geral-Setor de Ciências Biológicas e da Saúde, UEPG, 84030-900 Ponta Grossa-PR, $<$ dalva_rocha@uol.com.br>; ${ }^{3}$ Professor Doutor do Dep. de Botânica, Instituto de Biociências de Botucatu, UNESP, 18618-000 Botucatu-SP, <rodella@ibb.unesp.br>; ${ }^{4}$ Professor Adjunto do Dep. de Produção Vegetal, Faculdade de Ciências Agronômicas, UNESP, 18603-970 Botucatu-SP, <dmartins@fca.unesp.br>. 


\section{INTRODUÇÃO}

Dentre as espécies de Commelina (Commelinaceae), a mais importante é C. benghalensis, por infestar culturas em muitos países (Holm et al., 1977). Entretanto, existem outras plantas deste gênero (C. diffusa, C. erecta e C. villosa) que se encontram difundidas no território brasileiro e que também podem causar prejuízos econômicos nas culturas agrícolas, como soja, milho, pomares de laranja, cafezais, entre outras (Kissmann, 1997; Rocha et al., 2000).

No Brasil, essas quatro espécies são conhecidas popularmente como trapoeraba e são, geralmente, confundidas entre si. A identificação incorreta dessas espécies pode provocar dificuldades de controle químico, uma vez que cada uma apresenta comportamento distinto quanto aos herbicidas (Rocha et al., 2007), elevando os custos para o produtor, sendo, portanto, necessário identificar corretamente as espécies. Diferentes trapoerabas podem ocorrer simultaneamente, infestando a mesma cultura. Particularmente, nesses casos, a identificação das espécies é fundamental para a decisão em relação ao produto que deverá ser aplicado para controle. Assim, chaves que facilitem a identificação dessas espécies serão ferramentas muito importantes.

Métodos estatísticos multivariados, como a análise de agrupamento e a análise de componentes principais, têm sido utilizados em vários estudos para selecionar, com precisão, indicadores de similaridade entre grupos. Usando esses métodos, Peroni et al. (1999) avaliaram a diversidade intra e interespecífica de mandioca a partir da análise multivariada de 21 características morfológicas, possibilitando a classificação de etnovariedades amostradas em pequenas propriedades na região de Cananéia, na divisa entre os Estados de São Paulo e Paraná. Melo Filho et al. (2000) também classificaram 11 acessos interespecíficos de um banco de germoplasma de cará.

O método de análise de componentes principais foi aplicado por Santos et al. (2000) para classificar genótipos de amendoim, utilizando 23 características de natureza diversa, sendo 11 morfológicas, nove agronômicas e três protéico-enzimáticas. Rodella et al. (2006) e Costa et al. (2006) também usaram a análise de agrupamento e a análise de componentes principais como ferramenta na diferenciação entre plantas daninhas aquáticas.

De acordo com Moita Neto (2004), a análise de agrupamento e a análise de componentes principais são os dois métodos que podem fornecer informações estatísticas, permitindo selecionar indicadores com valor discriminatório para a segura identificação dessas espécies.

O presente trabalho teve como objetivo selecionar características morfológicas que possibilitem facilitar a identificação de C. benghalensis, C. villosa, C. diffusa e C. erecta, utilizando a técnica de análise multivariada.

\section{MATERIAL E MÉTODOS}

Exemplares de quatro espécies de trapoeraba (C. benghalensis, C. villosa, C. diffusa e C. erecta) foram amostrados em três áreas agrícolas de nove regiões do Estado de São Paulo e seis do Paraná (Figura 1). A seguir, foram cultivados em condições de casa de vegetação, no Departamento de Botânica do Instituto de Biociências de Botucatu, da Universidade Estadual Paulista (UNESP), em Botucatu-SP. As exsicatas estão registradas no Herbário BOTU, da Universidade Estadual Paulista, em Botucatu-SP, sob os números de 22990 a 23030.

As características morfológicas das plantas in vivo foram observadas com auxilio de estereomicroscópio e as mensurações foram feitas com dez repetições, utilizando-se paquímetro. Destacaram-se 25 características que evidenciam diferenças entre as espécies. Dessas, 12 são características morfológicas descritivas: hábito da planta, pilosidade do caule, pilosidade da folha, tipo de folha, formato do limbo (Hickey, 1973), ocorrência de aurícula na base do limbo, formato da antera no estame central, coloração dos filetes, formato do ápice da espata, ocorrência de soldadura posterior da espata, coloração do pólen dos estames laterais e desenvolvimento da terceira pétala. Outras 13 características quantitativas também foram avaliadas: comprimento e largura da terceira folha; comprimento e largura de sépala e pétala; comprimentos da espata, do pedúnculo, do estame central, dos estames laterais 


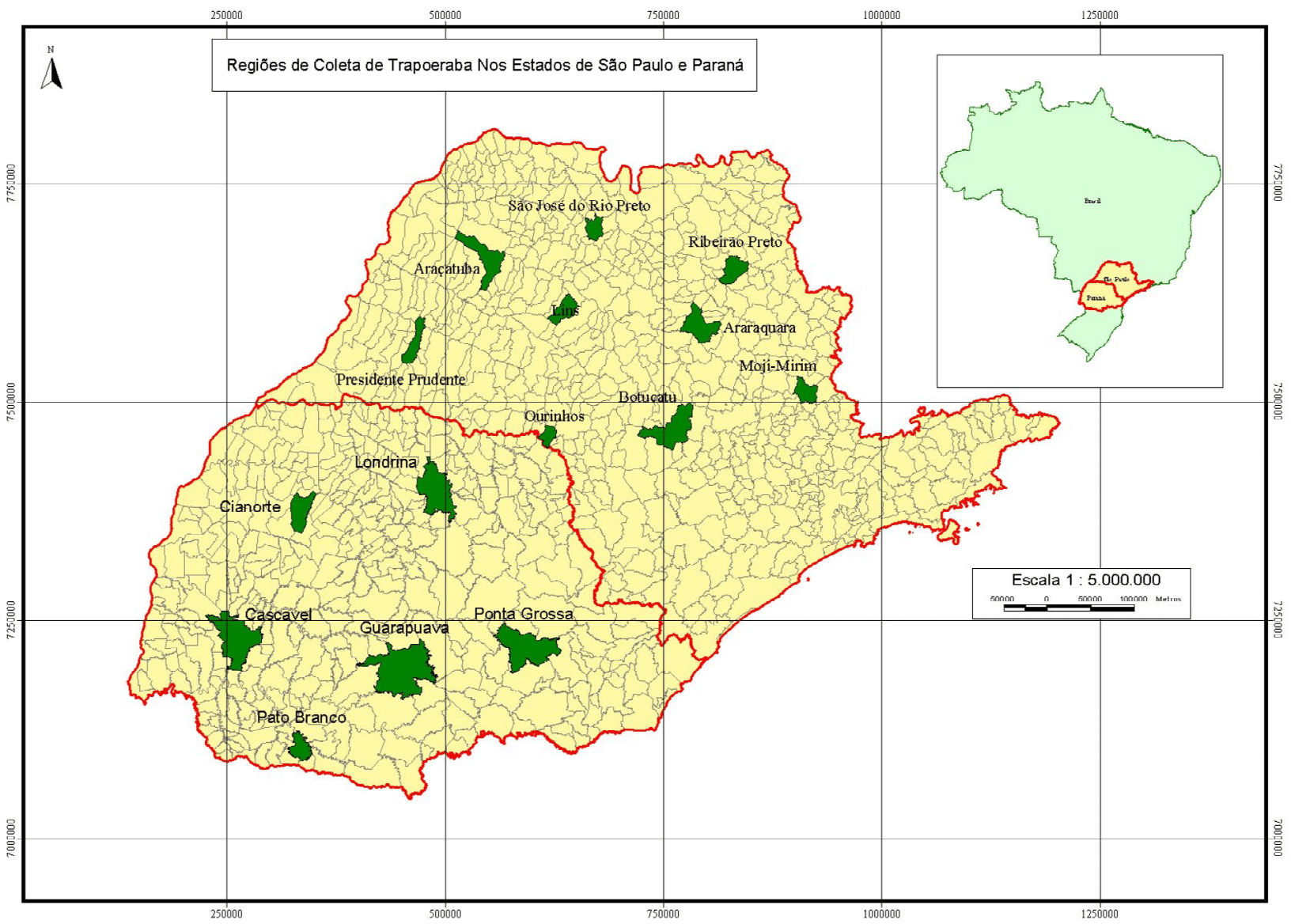

Figura 1 - Regiões de amostragem das quatro espécies de Commelina, localizadas nos Estados de São Paulo e Paraná, 2001.

e dos estaminódios; número de inflorescência por espata; e número de flores por inflorescência.

A análise estatística foi empregada separadamente sobre caracteristicas descritivas e quantitativas. Os métodos estatísticos de análise multivariada aplicados foram análise de agrupamento e análise de componentes principais (Sneath \& Sokal, 1973).

Com relação à análise de agrupamento das características descritivas, estas foram transformadas em valores numéricos e foi aplicado o coeficiente de similaridade "simple matching" para o conjunto de indicadores. Para análise de agrupamento das caracteristicas quantitativas, os dados foram transformados e, em seguida, empregou-se a distância euclidiana média para o conjunto dos indicadores. Tanto para as características descritivas como para as quantitativas, utilizou-se o algoritmo UPGMA, conforme recomendado por Rohlf (1970), com a finalidade de agrupar as espécies de Commelina conforme o grau de similaridade.

A análise de componentes principais, realizada com os valores médios referentes às características quantitativas, de acordo com Morrison (1967), foi empregada para verificar a capacidade discriminatória das características originais no processo de transformação dos agrupamentos; assim, essa análise reduz o conjunto dos indicadores a duas novas variáveis não correlacionadas, chamadas componentes principais $\left(\mathrm{Y}_{1}\right.$ e $\left.\mathrm{Y}_{2}\right)$. O resultado dessa análise também foi apresentado na forma de dispersão gráfica, para ilustrar o posicionamento relativo das quatro espécies. 


\section{RESULTADOS E DISCUSSÃO}

\section{Características morfológicas descritivas}

A Tabela 1 apresenta as 12 características morfológicas descritivas observadas para diferenciar as quatro espécies de Commelina. O dendrograma apresentado na Figura 2, resultante da análise de agrupamento dessas características, favorece a visualização da dissimilaridade entre essas espécies. Observa-se que a formação do primeiro agrupamento inclui C. benghalensis e C. villosa, ocorrendo aproximadamente a 0,5 na escala de distância euclidiana. Já $C$. erecta apresenta maior similaridade com $C$. benghalensis e $C$. villosa que C. diffusa, pois esta última constitui um grupo monoespecífico a aproximadamente 0,33 da referida escala, indicando possuir alto grau de dissimilaridade em relação às outras três espécies.

Constatou-se que, entre essas características descritivas analisadas, oito possuem alto valor taxonômico: hábito da planta, pilosidade do caule e da folha, formato do limbo, ocorrência de aurícula no limbo, formato do ápice da espata, coloração dos filetes e formato da antera do estame central.

Essas oito características selecionadas foram utilizadas na elaboração da chave de identificação para as espécies estudadas.
Chave de identificação para as espécies de Commelina, utilizando-se das características morfológicas descritivas:

1. Planta ereta; estame central com antera auricular; filetes translúcidos; espata fechada com ápice agudo; corola com uma pétala atrofiada (reduzida) e duas expandidas.

2. Caule e folha pouco pilosos; folha peciolada, eliptica-estreita, com auricula no limbo; pólen branco nos estames laterais ... C. erecta

2. Caule e folha intensamente pilosos; folha séssil, oblonga-estreita, sem aurícula no limbo; pólen amarelo nos estames laterais . C. villosa

1. Planta decumbente; folha peciolada, sem aurícula no limbo; estame central com antera sagitada.

3. Caule e folha intensamente pilosos; folha eliptica; espata fechada com ápice agudo; corola com uma pétala atrofiada (reduzida) e duas expandidas; filetes roxos; pólen branco nos estames laterais

C. benghalensis

3. Caule e folha pouco pilosos; folha eliptica-estreita; espata aberta com ápice acuminado; corola com três pétalas expandidas; filetes translúcidos; pólen amarelo nos estames laterais

C. diffusa

Rodella et al. (2006) também utilizaram análises estatísticas para diferenciar oito

Tabela 1 - Características morfológicas descritivas para diferenciação de quatro espécies de Commelina. Botucatu-SP, 2001

\begin{tabular}{|l|c|c|c|c|}
\hline \multicolumn{1}{|c|}{ Característica } & COMBE & COMVI & COMDI & COMER \\
\hline Hábito da planta & decumbente & ereto & decumbente & ereto \\
\hline Pilosidade do caule & intensa & intensa & escassa & escassa \\
\hline Pilosidade da folha & intensa & intensa & escassa & escassa \\
\hline Tipo da folha & peciolada & séssil & peciolada & peciolada \\
\hline Formato do limbo foliar & elíptica & oblonga-estreita & elíptica-estreita & elíptica-estreita \\
\hline Aurícula no limbo foliar & ausente & ausente & ausente & presente \\
\hline Formato da antera do estame central & sagitada & auricular & sagitada & auricular \\
\hline Coloração dos filetes & roxo & translúcido & translúcido & translúcido \\
\hline Formato do ápice da espata & aguda & aguda & acuminada & aguda \\
\hline Soldadura posterior da espata & fechada & fechada & aberta & fechada \\
\hline Coloração do pólen dos estames laterais & branco & amarelo & amarelo & branco \\
\hline Desenvolvimento da terceira pétala & reduzido & reduzido & expandido & reduzido \\
\hline
\end{tabular}

COMBE: C. benghalensis; COMVI: C. villosa; COMDI: C. diffusa; COMER: C. erecta. 


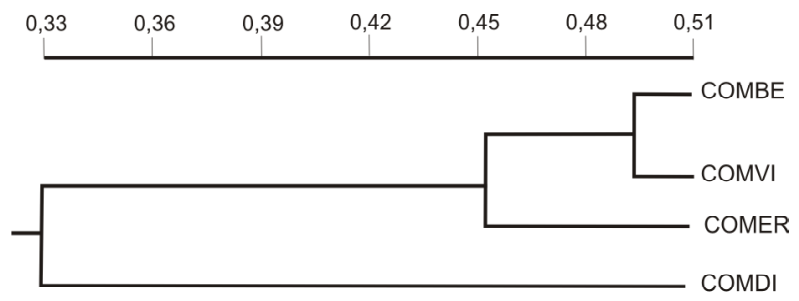

Figura 2 - Dendrograma resultante da análise de agrupamento das 12 características morfológicas descritivas de Commelina benghalensis (COMBE), C. villosa (COMVI), C. diffusa (COMDI) e C. erecta (COMER), utilizando-se o coeficiente de similaridade "simple matching". BotucatuSP, 2001

acessos de Egeria densae de E. najas, espécies estas daninhas aquáticas que ocorrem em reservatórios de usinas hidrelétricas do complexo da CESP, no Estado de São Paulo, obtendo resultados satisfatórios com a análise de apenas sete características anatômicas da lâmina foliar.

\section{Caracteristicas morfológicas quantitativas}

A Tabela 2 apresenta os valores médios das 13 características morfológicas quantitativas para cada uma das quatro espécies estudadas, que foram empregados na análise estatística multivariada. Na Tabela 3 estão apresentados os coeficientes de correlação entre as 13 características quantitativas e os dois primeiros componentes principais $\left(\mathrm{Y}_{1}\right.$ e $\left.\mathrm{Y}_{2}\right)$. A intensidade da contribuição dessas características para a discriminação das espécies está diretamente relacionada com os maiores valores absolutos dos componentes $\mathrm{Y}_{1}$ e $\mathrm{Y}_{2}$. A informação retida no conjunto dessas 13 características referentes ao primeiro componente foi de $62,63 \%$. As oito características que mais contribuíram para a discriminação das espécies de Commelina foram: comprimento dos estames laterais, da sépala, do estame central, dos estaminódios, da terceira folha, da espata, da pétala, e largura da sépala.

A informação retida para o segundo componente foi de $33,64 \%$, e os valores indicam outra ordem das variáveis que mais contribuíram para a distinção das quatro espécies de Commelina: número de inflorescência por espata, número de flores por inflorescência, comprimento do pedúnculo, largura da pétala e largura da terceira folha (Tabela 3). No conjunto, os componentes $\mathrm{Y}_{1}$ e $\mathrm{Y}_{2}$ revelaram confiabilidade de $96,27 \%$, possibilitando realizar a seleção das características com maior valor taxonômico (Tabela 3).

Trabalhos que empregaram essas análises estatísticas para separar grupos intra ou

Tabela 2 - Valores médios das características morfológicas quantitativas para diferenciação de quatro espécies de Commelina. Botucatu-SP, 2001

\begin{tabular}{|l|c|c|c|c|}
\hline \multicolumn{1}{|c|}{ Característica } & COMBE & COMVI & COMDI & COMER \\
\hline Comprimento (cm) da terceira folha & 4,17 & 9,76 & 3,19 & 6,19 \\
\hline I argura (cm) da terceira folha & 1,92 & 3,26 & 1,14 & 1,74 \\
\hline Comprimento (cm) da sépala & 0,30 & 0,49 & 0,30 & 0,54 \\
\hline Largura (cm) da sépala & 0,21 & 0,35 & 0,21 & 0,43 \\
\hline Comprimento (cm) da pétala & 0,47 & 0,70 & 0,41 & 1,05 \\
\hline Largura (cm) da pétala & 0,60 & 0,89 & 0,54 & 1,52 \\
\hline Comprimento (cm) da espata & 1,10 & 1,98 & 1,45 & 1,79 \\
\hline Comprimento (cm) do pedúnculo & 1,85 & 2,52 & 1,55 & 1,37 \\
\hline Comprimento (cm) estame central & 0,42 & 0,64 & 0,42 & 0,53 \\
\hline Comprimento (cm) cstames latcrais & 0,57 & 0,92 & 0,48 & 0,81 \\
\hline Comprimento (cm) estaminódios & 0,30 & 0,47 & 0,36 & 0,46 \\
\hline Número de inflorescência/espata & 2,00 & 2,50 & 2,50 & 1,00 \\
\hline Número de flores/inflorescência & 3,10 & 9,20 & 3,90 & 3,80 \\
\hline
\end{tabular}

COMBE: C. benghalensis; COMVI: C. villosa; COMDI: C. diffusa; COMER: C. erecta.

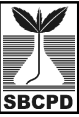


Tabela 3 - Correlações entre as 13 características morfológicas quantitativas das quatro espécies de Commelina e os dois primeiros componentes principais $\left(\mathrm{Y}_{1}\right.$ e $\left.\mathrm{Y}_{2}\right)$. Porcentagem de informação retida e acumulada em $\mathrm{Y}_{1}$ e $\mathrm{Y}_{2}$ e ordenação das características quanto ao seu poder discriminatório - indicada entre parênteses. Botucatu-SP, 2001

\begin{tabular}{|l|c|c|}
\hline \multicolumn{1}{|c|}{ Característica } & $\mathrm{Y}_{1}$ & $\mathrm{Y}_{2}$ \\
\hline Comprimento da terceira folha & $0,9126(5)$ & $0,3985(8)$ \\
\hline Largura da terceira folha & $0,7220(10)$ & $0,6101(5)$ \\
\hline Comprimento da sépala & $0,9598(2)$ & $-0,2733(10)$ \\
\hline Largura da sépala & $0,9115(6)$ & $-0,4062(7)$ \\
\hline Comprimento da pétala & $0,8073(8)$ & $-0,5897(6)$ \\
\hline Largura da pétala & $0,7480(9)$ & $-0,6637(4)$ \\
\hline Comprimento da espata & $0,9008(7)$ & $0,1036(12)$ \\
\hline Comprimento do pedúnculo & $0,3988(12)$ & $0,8856(2)$ \\
\hline Comprimento do estame central & $0,9458(3)$ & $0,3208(9)$ \\
\hline Comprimento dos estames laterais & $0,9869(1)$ & $0,1050(11)$ \\
\hline Comprimento dos estaminódios & $0,9323(4)$ & $-0,0521(13)$ \\
\hline Númcro de inflorescência por cspata & $-0,3229(13)$ & $0,9129(1)$ \\
Número de flores por inflorescência & $0,6910(11)$ & $0,7086(3)$ \\
Informação retida (\%) & 62,63 & 33,64 \\
\hline Informação acumulada (\%) & 62,63 & 96,27 \\
\hline
\end{tabular}

interespecíficos não obtiveram índice de confiabilidade tão alto quanto este obtido no presente trabalho. Melo Filho et al. (2000) constataram $89,12 \%$ de informação acumulada para os dois componentes principais, avaliando 14 descritores de 11 acessos de cará. Rodella et al. (2006) verificaram confiabilidade de $83,94 \%$ para os dois primeiros componentes principais, a partir da análise de apenas sete características anatômicas foliares de oito acessos de duas espécies de Egeria. Costa et al. (2006) também obtiveram $81,98 \%$ de informação acumulada pelos dois primeiros componentes principais, analisando 19 características anatômicas quantitativas de folhas para distinguir sete espécies de diferentes gêneros de plantas daninhas aquáticas.

Para as quatro espécies de Commelina estudadas, a análise de agrupamento e a análise de componentes principais das características quantitativas revelaram a formação de três grupos (Figuras 3 e 4). No entanto, a formação desses grupos difere daquela mostrada pelo dendrograma resultante da análise das características descritivas. O primeiro grupo formado pela análise das características quantitati- vas foi constituído por $C$. benghalensis e C. diffusa, enquanto o segundo e o terceiro grupo são monoespecíficos formados por C. villosa e $C$. erecta, respectivamente. Estas duas últimas espécies apresentam o maior grau de dissimilaridade.

Os dados estatísticos revelaram que $C$. benghalensis e $C$. diffusa apresentaram os menores valores para o tamanho dos estames laterais, do estame central, dos estaminódios, da sépala, da pétala e da terceira folha; para C. villosa e $C$. erecta, os maiores valores foram constatados para o comprimento dos estames laterais e da terceira folha. Isso indica que as flores de $C$. villosa e $C$. erecta são de tamanho maior que as de $C$. benghalensis e $C$. diffusa.

Ressalta-se ainda que $C$. benghalensis, C. diffusa e C. villosa apresentaram os maiores valores de número de inflorescência por espata e comprimento do pedúnculo, o que as colocam distantes de $C$. erecta (Figuras 3 e 4).

Dessa forma, uma segunda chave de identificação para as quatro espécies de Commelina foi também elaborada, considerando apenas as características morfológicas quantitativas. 


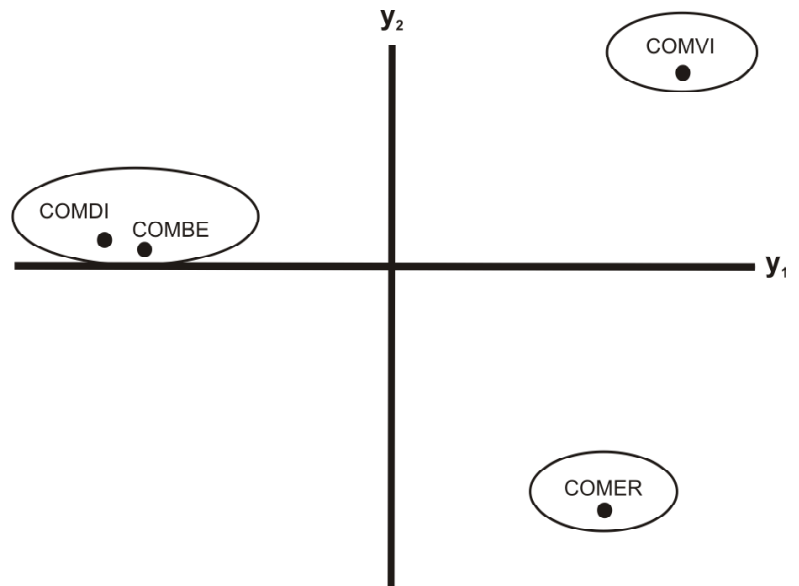

Figura 3 - Dispersão gráfica de Commelina benghalensis (COMBE), C. villosa (COMVI), C. diffusa (COMDI) e C. erecta (COMER), utilizando os do is primeiros componentes principais $\left(\mathrm{Y}_{1}\right.$ e $\left.\mathrm{Y}_{2}\right)$ para o conjunto das 13 características morfológicas quantitativas. Botucatu-SP, 2001.

Chave de identificação para as espécies de Commelina, utilizando-se das caracteristicas morfológicas quantitativas:

1. Comprimento médio da terceira folha maior que $8,0 \mathrm{~cm}$; comprimento médio do estame central maior que $0,6 \mathrm{~cm}$; mais de oito flores por inflorescência

C. villosa

1. Comprimento médio da terceira folha menor que $8,0 \mathrm{~cm}$; comprimento médio do estame central menor que $0,6 \mathrm{~cm}$; menos de cinco flores por inflorescência.

2. Comprimento médio da terceira folha aproximadamente $6,2 \mathrm{~cm}$; comprimento médio da sépala maior que $0,5 \mathrm{~cm}$; largura média da sépala maior que $0,4 \mathrm{~cm}$; comprimento médio da pétala maior que $0,9 \mathrm{~cm}$; largura média da pétala maior que $1,2 \mathrm{~cm}$

C. erecta

2. Comprimento médio da sépala menor que $0,4 \mathrm{~cm}$; largura média da sépala menor que $0,3 \mathrm{~cm}$; comprimento médio da pétala menor que $0,6 \mathrm{~cm}$; largura média da pétala menor que $0,7 \mathrm{~cm}$.

3. Comprimento médio da terceira folha aproximadamente $4,2 \mathrm{~cm}$; média de 3,1 flores por inflorescência; média de 2,0 inflorescências por espata

C. benghalensis

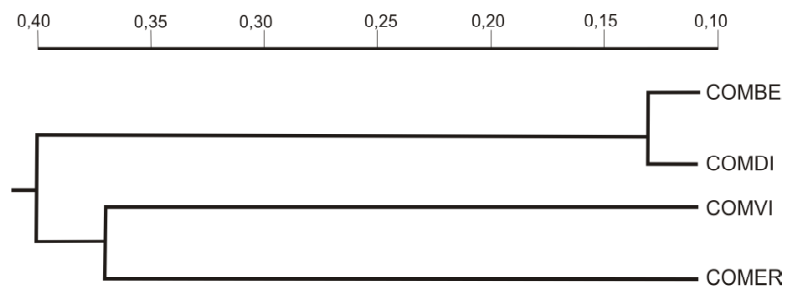

Figura 4 - Dendrograma resultante da análise de agrupamento das 13 características morfológicas quantitativas de Commelina benghalensis (COMBE), C. villosa (COMVI), C. diffusa (COMDI) e C. erecta (COMER), utilizando-se a distância euclidiana média. Botucatu-SP, 2001.

3. Comprimento médio da terceira folha aproximadamente $3,2 \mathrm{~cm}$; média de 3,9 flores por inflorescência; média de 2,5 inflorescências por espata

C. diffusa

O uso da chave de identificação tem a praticidade de comparar as plantas, enquanto os manuais de identificação de plantas daninhas apresentam apenas as descrições resumidas das espécies, como se observa nos trabalhos de Kissmann (1997) e Lorenzi (2000), dois manuais ilustrados muito utilizados para identificações de plantas daninhas.

Santos et al. (2000) ressaltam a importância do uso desta técnica como ferramenta para identificação criteriosa de populações intraespecíficas, contribuindo para o conhecimento dos diferentes genótipos de amendoim.

As análises estatísticas multivariadas utilizadas comprovaram o valor taxonômico das características morfológicas avaliadas, permitindo a elaboração de duas chaves de identificação para as quatro espécies de Commelina estudadas. A apresentação dessas características na forma de chave de identificação poderá facilitar o reconhecimento dessas espécies.

\section{LITERATURA CITADA}

COSTA, N. V.; RODELLA, R. A.; MARTINS, D. Diferenciação de espécies daninhas aquáticas pela análise multivariada de caracteres estruturais foliares. Planta Daninha, v. 24, n. 1, p. 13-20, 2006.

HICKEY, L. J. Classification of the architecture of dicotyledon ous leaves. Am. J. Bot., v. 60, n. 1, p. 17-33, 1973. 
HOLM, L. G. et al. The world's worst weeds distribution and biology. Honolulu: University Press, 1977. 609 p.

KISSMANN, K. G. Plantas infestantes e nocivas. 2.ed. São Paulo: BASF, 1997. Tomo 1. 824 p.

LORENZI, H. Plantas daninhas do Brasil. 3. ed. Nova Odessa: Instituto Plantarum, 2000. 339 p.

MELO FILHO, P. A. et al. Classificação de germoplasma de Dioscorea sp. através da análise de componentes principais. Ci. Rural, v. 30, n. 4, p. 619-624, 2000.

MOITA NETO, J. M. Estatística multivariada: uma visão didático-metodológica. R. Filos. Ens., v. 1, p. 1, 2004.

MORRISON, D. F. Multivariate statistical methods. New York: MacGrawn Hill, 1967. 338 p.

PERONI, N.; MARTINS, P. S.; AKIHIKO, A. Diversidade inter e intra-específica e uso da análise multivariada para morfologia da mandioca (Manihot esculenta Crantz): um estudo de caso. Sci. Agric., v. 56, n. 3, p. 587-595, 1999.
ROCHA, D. C. et al. Efeito de herbicidas sobre quatro espécies de trapoeraba. Planta Daninha, v. 25, n. 25, p. 359-364, 2007.

ROCHA, D. C.; RODELLA, R. A.; MARTINS D.

Ocorrência de Commelina villosa como planta daninha em áreas agrícolas no Estado do Paraná-PR, Brasil. Planta

Daninha, v. 18, n. 2, p. 161-167, 2000.

RODELLA, R. A. et al. Diferenciação entre Egeria densa e Egeria najas pelos caracteres anatômicos foliares. Planta Daninha, v. 24, n. 2, p. 211-220, 2006.

ROHLF, F. J. Adaptative hierarchical clustering schemes. Syst. Zool., v. 19, n. 1, p. 58-82, 1970

SANTOS, R. C. et al. Classificação de genótipos de amendoim baseada nos descritores agromorfológicos e isoenzimáticos. Ci. Rural, v. 30, n. 1, p. 55-59, 2000.

SNEATH, P. H. A.; SOKAL, R. R. Numerical taxonomy. San Francisco: W.H. Freeman, 1973. 573 p. 